Supporting Information

\title{
Sustainable Low-Cost Green Electrodes with High Volumetric Capacitance for Aqueous Symmetric Supercapacitors with High Energy Density
}

Qinxing Xie $^{a, *}$, Rongrong Bao ${ }^{a}$, Anran Zheng $^{a}$, Yufeng Zhang ${ }^{a}$, Shihua Wu ${ }^{b}$, Chao Xie ${ }^{c}$, Peng Zhao ${ }^{b}$

${ }^{a}$ Tianjin Key Laboratory of Fiber Modification and Functional Fibers, School of Materials Science and Engineering, Tianjin Polytechnic University, Tianjin 300387, China

${ }^{\mathrm{b}}$ Department of Chemistry, Nankai University, Tianjin 300017, China

${ }^{\mathrm{c}}$ School of Materials Science and Engineering, Shandong University of Technology, Zibo 255049, China 


\section{Preparation of graphite oxide}

Graphite oxide was prepared by a modified Hummers method. ${ }^{1}$ In a typical process, $2.0 \mathrm{~g}$ of natural graphite powders and $1.0 \mathrm{~g}$ of $\mathrm{NaNO}_{3}$ were successively mixed with $50 \mathrm{ml}$ of concentrated $\mathrm{H}_{2} \mathrm{SO}_{4}(98 \%)$ in a $500 \mathrm{ml}$ flask, and the mixture was stirred for $1 \mathrm{~h}$ in an ice-water bath. $6.0 \mathrm{~g}$ of $\mathrm{KMnO}_{4}$ was added slowly under vigorous stirring, and the temperature was maintained below $20^{\circ} \mathrm{C}$. After the removal of ice-water bath, the resulted mixture was further stirred for $3 \mathrm{~h}$ at $35{ }^{\circ} \mathrm{C}$. After that, $100 \mathrm{ml}$ of distilled water was added under vigorous stirring at a temperature of $98{ }^{\circ} \mathrm{C}$. The diluted suspension was further stirred for $15 \mathrm{~min}$, the reaction was then terminated by the addition of $280 \mathrm{ml}$ of distilled water. $30 \mathrm{ml}$ of $\mathrm{H}_{2} \mathrm{O}_{2}$ solution (30\%) was added under constant stirring till the solution color turned into bright-yellow. The suspension was filtrated, and the solid obtained was washed with 5\% hydrochloric acid and deionized water in turn for several times until no sulfate in filtrate could be detected. The solid was freeze-dried finally. 

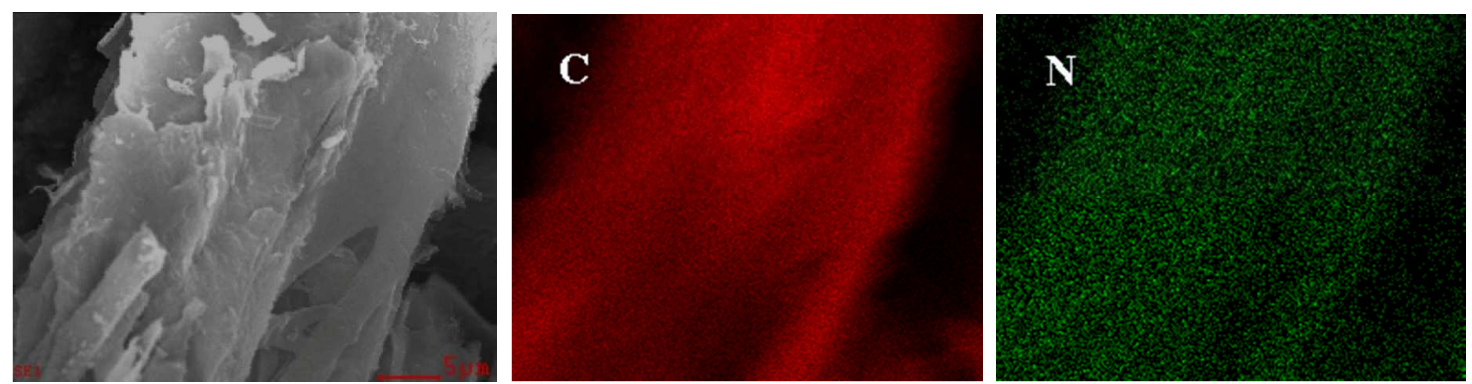

Figure S1. Elemental mapping of $\mathrm{C}$ and $\mathrm{N}$ in $\mathrm{N}-\mathrm{AC} / \mathrm{Gr} 1$ composite. 

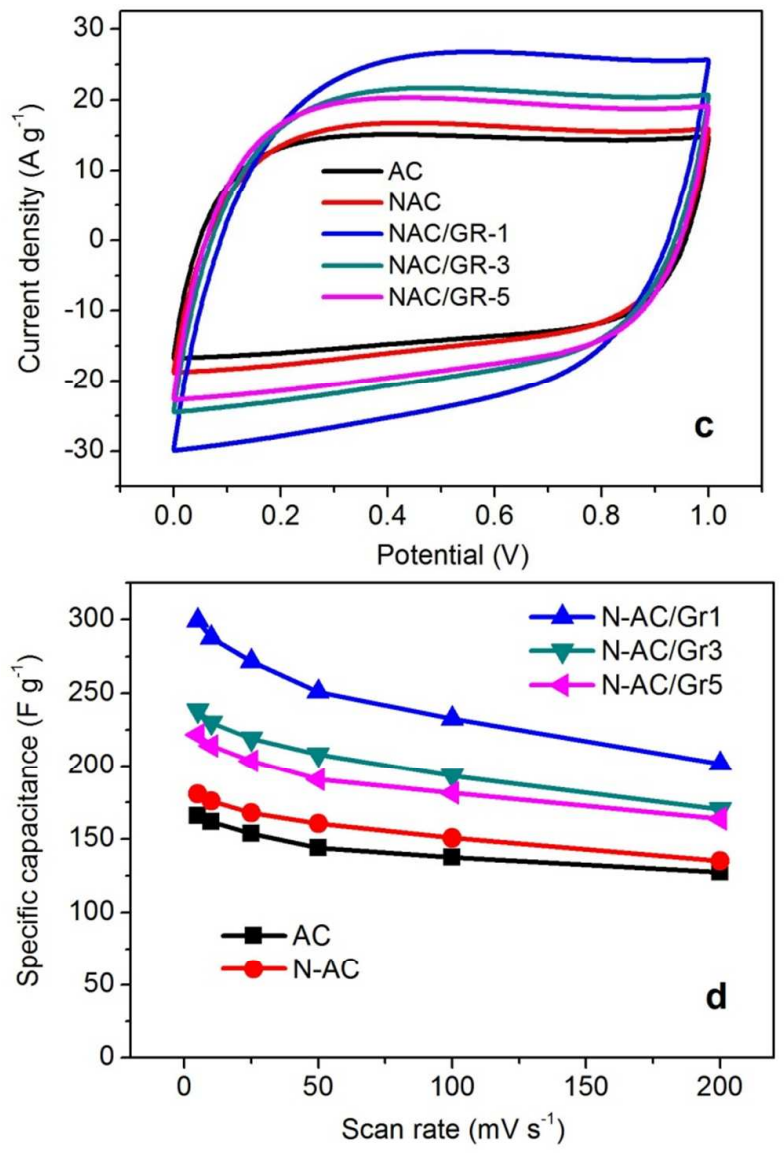

Figure S2. (a) A comparison of CV curves at a scan rate of $200 \mathrm{mV} \mathrm{s}-1$, (b) specific capacitances at various scan rates from 5 to $200 \mathrm{mV} \mathrm{s}^{-1}$ for the samples. 

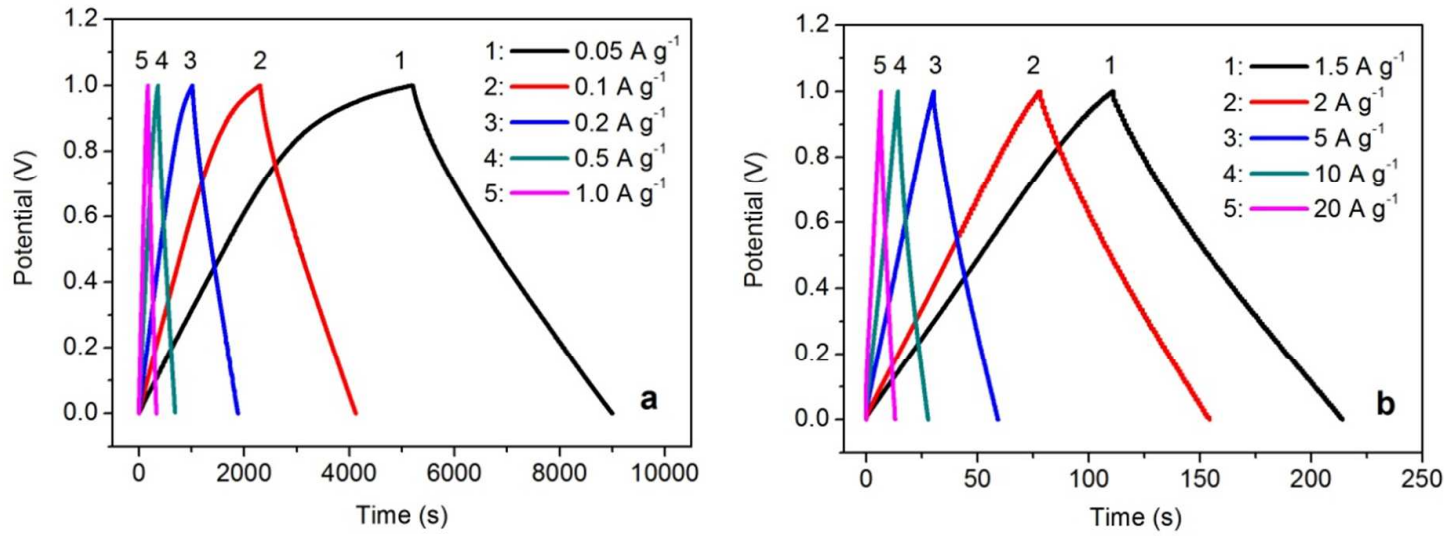

Figure S3. GCD curves of N-AC/Gr1 composite at current densities varied from 0.05 to $20 \mathrm{~A} \mathrm{~g}^{-1}$. 


\section{REFERENCES}

(1) Hummers, W. S.; Offeman, R. E., Preparation of Graphitic Oxide. J. Am. Chem. Soc. 1958, 80 (6), 1339-1339. 